\title{
Informações sobre a morbidade hospitalar em idosos nas internações do Sistema Único de Saúde do Brasil
}

\author{
Information on seniors' morbidity upon internments \\ on the Brazilian Unified Health System
}

Ana Luzia Batista de Góis ${ }^{1}$

Renato Peixoto Veras ${ }^{2}$

${ }^{1}$ FaculdadedeCiências M édicas, Universidade do Estado do Rio deJaneiro. Av. Prof. M anoel deAbreu 444/2음 andar, Vila I sabel. 20550-170 Rio de Janeiro RJ. anagoisfisio@globo.com ${ }^{2}$ U niversidade do Estado do Rio deJaneiro.
Abstract Considering the high hospital costs of the elderly, this study aimed to compare the seven highest frequencies of morbidity in the elderly using data from $\mathrm{H}$ ospital Information System. The methodology used was the evaluation of information obtained in the databaseDatasus/M inistry of $\mathrm{H}$ ealth on the morbidity of hospital admissions in the elderly, in 2005 and 1994. Additionally, it was performed the calculation of standard and adjusted rates by the direct standardization method using Epidat 3.1. The main results were the diseases of the circulatory system remained prevalent in $2005(28 \%)$ and 1994 (32\%), with a reduction of $4 \%$ between these years. The number of cases of neoplasms doubled from 1994 ( 4\%) to 2005 (8\%), and infectious and parasitic diseases were basically maintaining the same percentage of 7\% in 1994 and 2005. It can be concluded that in Brazil, the hospital morbidity in elderly remains the prevalence of cardiovascular diseases. At the same time, there is a reduction of non-infectious and parasitic diseases, and also signs of recent and sharp increase of neoplasms. It is recommended the use of comparison studies between periods become as a tool used in management.

Key words M orbidity, Elderly, Public health
Resumo Tendo em vista os altos custos hospitalares dos idosos, o presente estudo teve como obj etivo comparar as sete maiores frequências de morbidade hospitalar em idosos no ano de 2005 com o ano de 1994, através dos dados do Sistema de Informações H ospitalares (SIH /M S). M etodologia: estudo de avaliação das informaç̃os obtidas na base de dados do Datasus, do M inistério da Saúde, sobrea morbidade hospitalar das internações em idosos, nos anos de 2005 e de 1994. Adicionalmente, procedeu-se ao cálculo das taxas padrão e ajustadas, pelo método direto de padronização com o uso do programa Epidat 3.1. Principais resultados: as doenças do aparelho circulatório se mantiveram predominantesem 2005 (28\%) eem 1994 (32\%), com uma redução de 4\% entre estes anos; houve 0 aumento em dobro das neoplasias de 1994 (4\%) para 2005 (8\%), e as doenças infecciosas e parasitárias apresentaram a manutenção basicamentedo mesmo percentual de 7\%, em 1994 eem 2005. Pode-se concluir que, no Brasil, a morbidade hospitalar em idosos mantém o predomínio das doenças do aparelho circulatório, ao mesmo tempo que se observa a não redução das doenças infecciosas e parasitárias; e ainda sinaliza-se 0 aumento recente e acentuado das neoplasias. Recomenda-se que os estudos decomparações entre períodos sejam umas das ferramentas de uso na gestão.

Palavras-chave M orbidade, Idoso, Saúde pública 
Introdução

0 aumento da população idosa é um fenômeno observado em diversos países, inclusive no Brasil. 0 atual perfil epidemiológico das doenças crônicas que acometem essa faixa da população leva consequentemente ao aumento da improdutividade dos indivíduos e ao aumento dos custos governamentais. Porém, em se tratando de saúde pública, o Brasil ainda tem como agravante os números elevados do perfil epidemiológico anterior (doenças infectocontagiosas) somados a doenças emergentes.

A informação sobre a comparação de taxas de morbidades hospitalares nos anos de $1994 \mathrm{e}$ 2005 permite melhor visibilidade do quadro de morbidade atual dos idosos no país. Assim, 0 presente trabalho poderá fornecer subsídios que auxiliarão o planejamento das prioridades e escolhas nas atenções em saúde pública voltadas para os idosos.

\section{Desenvolvimento}

No Brasil, são consideradas idosas as pessoas com mais de 60 anos. Esta idade também é usada como delimitador pela Organização Mundial da Saúde (OMS) ${ }^{1}$. De acordo com o Instituto Brasileiro de Geografia e Estatística (IBGE)², 0 número de idosos em nosso país é um dos maiores do mundo - 14,5 milhões de pessoas com mais de 60 anos, em 2000 - e deverá multiplicarse por cinco até 2025, assumindo-se os pressupostos de grandes pesquisadores ${ }^{3-6}$.

A transição epidemiológica apresenta um quadro de morbimortalidade em que as doenças cardiovasculares representam atualmente mais de $40 \%$ das mortes registradas no país ${ }^{2}$. 0 Brasil apresenta a segunda maior taxa de mortalidade por acidente vascular cerebral (AVC) no mundo, e esta é a maior causa de morte para os brasileiros acima de 65 anos $^{7}$. Pesquisa realizada por Karsch ${ }^{8}$ apontou que o Brasil apresenta a segunda maior taxa de mortalidade por acidente vascular cerebral no mundo, equeo AVC constitui a maior causa de morte para homens e mulheres brasileiros acima de 65 anos.

Segundo Camarano $^{9}$, as duas causas mais frequentes de internação, para ambos os sexos, são a insuficiência cardíaca e coronariana e as doenças pulmonares, que se revezam como a primeira e a segunda causas. O AVC agudo, a crisehipertensiva, as enteroinfecções, a desnutrição, a desidratação e a anemia estão sempre pre sentes como causas intermediárias. Porém, os acometimentos do envelhecimento não devem ser explicados por uma única doença.

O idoso consome mais serviços de saúde, as internações hospitalares são mais frequentes e 0 tempo de ocupação do leito é maior do que o de outras fai xas etárias. Em geral, as doenças dos idosos perduram por vários anos e exigem acompanhamento médico e de equipes multidisciplinares permanentes, além de intervenções contínuas ${ }^{6}$.

Carvalho-Filho ${ }^{10}$ evidenciou que a iatrogenia é frequente em pacientes idosos hospitalizados, podendo determinar manifestações graves, emesmo fatais - sendo encontrada também nas instituições de longa permanência, cujo perfil atual é deuma população cada vez maisidosa, mais doenteemaisfuncionalmentedependente. N os hospitais, pode-se encontrar eventos adversos em cerca de $4 \%$ dos pacientes internados. Um estudo hospitalar citado por Rothschild, Bates e Leape ${ }^{11}$ demonstrou que a taxa de complicações hospitalares para os idosos é cerca de 1,5 vez maior que os do grupo etário mais jovem.

Os idosos, as crianças e os portadores de deficiência são as maiores vítimas de maus-tratos e negligência nos cuidados, cujas principais consequências são as quedas, as contenções físicas, as úlceras de decúbito, as infecções hospital ares e as complica-ções perioperatórias ${ }^{12}$. Nos idosos, a incidência de quedas é de cerca de $32 \%$ ao ano, sendo que $24 \%$ resultam em injúrias que requerem atendimento médico. Em idosos institucionalizados, a incidência chega a $50 \%$ ao ano. Desses pacien-tes, cerca de $25 \%$ morrem dentro de um ano, $15 \%$ são institucionalizados e $60 \%$ nunca recuperam o nível funcional prévio ${ }^{11}$. Os estudos mostram que idosos que já caíram anteriormente têm de quatro a vinte vezes mais chance de sofrer uma fratura ${ }^{13}$. A adoção de grades protetoras nos leitos, o bloqueio de acesso a escadas eo uso de pisos antiderrapantes têm reduzido bastante a incidência de quedas. Aquel es que sofrem quedas apresentam um grande declínio funcional nas atividades de vida diária enas atividades sociais, com aumento do risco de institucional izaçãa ${ }^{14}$.

Os idosos são particularmente suscetíveis a infecções nosocomiais, princi-palmente respiratórias, urinárias e de pele. Um estudo retrospectivo de 1.200 infec-ções nosocomiais ${ }^{15}$ encontrou um risco dez vezes maior entre o grupo etário de 70 a 79 anos do que entre o grupo de 40 a 49 anos. Em instituições asilares, o problema pode chegar a proporções ainda maiores, pela fragilidade dos residentes e a frequente superl otação dos quartos, nem sempre arejados. A proxima- 
damente $5 \%$ dos pacientes hospitalizados adquirem úlceras de decúbito durante a internaçãa ${ }^{11}$.

$N$ as instituições de longa perma-nência, um estudo norte-americano que envolveu 51 casas de repouso e quase vinte mil residentes revelou que $11 \%$ dos idosos já tinham úlceras na admissão ${ }^{16}$. A incidência de novas escaras em um ano foi de $13 \%$, epacientes diabéticos têm aproximadamente três vezes mais risco de adquirirem es$\operatorname{caras}^{17}$. Zinco e vitaminas A e $E$ são elementos que frequentemente estão ausentes na dieta de pacientes crônicos e que podem contribuir para dificuldade de cicatrização de feridas ${ }^{18}$.

A infecção do trato urinário (ITU) é das mais comuns no homem com mais de 65 anos, podendo levar à internação hospitalar, com grandes preju-ízos para o paciente. A bactéria que mais comumente causa o ITU é chamada Escherichia coli, responsável por $80 \%$ a $85 \%$ dos casos. Doenças da próstata, prolapso vesical ( "queda da bexiga"), presença de cálculos urinários e as doenças que causam disfunção da bexiga como na sequela de AVE, demências, diabetes mellitus, dentre outras, podem contribuir para o aparecimento da infecção urinária. A presença de incontinên-cia urinária também pode contribuir para a infecção urinária, devido ao uso de sonda vesical, predispondo à infecção. No entanto, a própria hospitalização frequente pode levar à contaminação por bactérias mais resistentes aos antibióticos, evoluindo para um quadro mais grave ${ }^{19}$.

As alterações pulmonares que fazem parte do processo de envelhecimento normal, tais como a redução da elasticidade da musculatura intercostal e a redução da capacidade vital, favorecem 0 aumento de "bactérias" e o perigo de uma pneumonia. Existe uma redução dos mecanismos de defesa, por isso é tão importante a vacinação do idoso contra a gripe e a pneumonia. No idoso, 0 comprometimento respiratório pode estar relacionado ao sedentarismo; como não necessitam de adaptação ventilatória ao esforço, os idosos não percebem as dificuldades respiratórias e, quando internados, esse quadro tende a se agravar ${ }^{20,21}$.

As doenças do trato gastrintestinal nos idosos são influenciadas pelas alterações do processo digestivo. Dentre estas, destacamos as alterações dentárias, as relativas ao mecanismo de deglutição, redução das secreções gástricas e enzimáticas, as relativas à redução do tônus da musculatura intestinal e a lentificação nos reflexos ${ }^{22}$. 0 resultado éuma tendência ao engasgamento, $\mathrm{e}$ quando isso ocorre pode provocar infecção, le vando a uma pneumonia. Há também problemasna absorção de nutrientes, e observa-se uma peristalse mais lenta; nos idosos restritos ao leito, a peristalse é mais lenta ainda. Além disso, eles tendem a ingerir menor quantidade de alimentos, favorecendo a constipação intestinal ${ }^{23}$.

Cerca de metade a três quartos das mortes por causas cirúrgicas referem-se a idosos ${ }^{11}$. Isto se deve à maior comorbidade que eles apresentam e também à apresentação atípica de situações de emergência, retar-dando o diagnóstico. As cirurgias de urgência em geriatria têm uma mortalidade de $21 \%$, enquanto as eletivas levam à morte em apenas $1,9 \%$ dos $\operatorname{casos}^{24}$. N esse sentido, o retardo numa indicação cirúrgica pode, muitas vezes, constituir uma iatrogenia ${ }^{19}$.

Os efeitos colaterais de drogas são o tipo mais comum de eventos adversos em idosos. Segundo Doucet et al. ${ }^{25}, 20 \%$ dos casos requerem hospitalização. Em instituições, a prevalência pode chegar a 32\%, muitas vezes pela falta de reavaliação da terapêutica inicialmente instituída ${ }^{26}$. Outra causa frequentede iatrogenia éa prescrição pouco clara. Um estudo norte-americano relatou uma diminuição de $55 \%$ dos erros na administração de medicamentos quando a prescrição foi computadorizada ${ }^{27}$.

0 câncer, na maioria dos casos, ocorre após os 60 anos. Essa maior incidência no idoso se deve ao fato de que cerca de $80 \%$ de todos os cânceres estão relacionados, direta ou indiretamente, ao tempo de exposição a agentes cancerígenos, sendo, atualmente, a segunda causa de morte no Brasil, logo após as doenças cardiovasculares ${ }^{28}$. Os órgãos mais comumente atingidos, por ordem de frequência, são: no homem, a pele, a próstata, o pulmão, o estômago e 0 intestino; na mulher, a mama, a pele, o colo do útero, o intestino, o estômago eo pulmão; além do sangue e do sistema linfático (leucemias elinfomas) em ambos os sexos ${ }^{29}$.

Segundo Camarano ${ }^{9}$, a taxa de utilização da internação na fai xa de 80 e mais anos étrês vezes maior quea da faixa etária de 55 a 59 anos. Quanto à questão dos custos, verificou-se que o custo médio mais elevado ocorrena faixa etária de 60 a 69 anos, tornando-se decrescentes com a idade, revelando que, pelo menos no que se refere ao SistemaÚ nico de Saúde (SUS), a el evação do custo com atenção médico-hospitalar aos idosos não está relacionada ao aumento do custo dos procedimentos. A elevação de custos está relacionada à taxa de utilização, e as medidas de saúde pública que objetivem melhor atenção ao idoso não precisam, necessariamente, elevar as despesas com saúde, mas sim substituir a quantidade de procedimentos de internação pela qualidade 
desse serviço, reduzindo assim as taxas de utilização, de forma queo paciente voltemenos vezes ao hospital. Alternativas concretas para a realização desse objetivo podem ser os programas de saúde em domicílio e a internação domiciliar.

Pelos dados apresentados, fica evidente $0 \mathrm{im}$ pacto queo envelhecimento da população brasileira produz no setor de saúde, principalmente no sistema hospitalar. Os gestores de saúde no país devem estar atentos para esse fato e para 0 crescimento da população que estará envelhe cendo nas quatro próximas décadas. Isso representa um grande desafio para o sistema de saúde, que poderá ter seus leitos hospitalares bloqueados e, pior, sem responder adequadamente às necessidades de saúde dessa população. Acrescentam-se a esse desafio o pequeno número de profissionais de saúde capacitados para 0 atendimento de idosos (geriatras e gerontólogos) ea falta da fisioterapia como prioridade dentre as alternativas eficientes ao atendimento à saúde dessa população.

Entre os novos modelos que vêm sendo discutidos para o enfrentamento desses problemas, ganham destaque as propostas de desospitalização e a ampliação do uso da assistência domici$\operatorname{liar}^{30}$. Rebelatto e M orelli ${ }^{14}$ sugerem que, em se tratando de reabilitação, a fisioterapia mais intensa seja instituída precocementena internação hospitalar, para ser mais efetiva e estendida para o domicílio, sem interrupção. O denheimer ${ }^{31} \mathrm{e}$ Góis ${ }^{32}$ afirmam que muitos dos declínios neurológicos geriátricos podem ser revertidos ou estabilizados com a fisioterapia precoce.

O Sistema Ú nico de Saúde apresenta atualmente como estratégia o Plano N acional de Saúde, que está expresso de modo a objetivar as intenções e os resultados a serem buscados no período de 2004 a 2007, traduzidos em objetivos, diretrizes e metas que possam responder às necessidades da população e que se constituam como referência para os gestores e para o controle social do próprio SUS. Cabe ressaltar também que, para a operacionalização do Plano $\mathrm{N}$ acional, são fundamentais as definições das responsabilidades de cada ente governamental, de cada nível do sistema e de cada serviço, de modo a se produzirem ações integrais consoantes com as necessidades das respectivas populações e de promoção da equidade social ${ }^{33}$.

0 presente estudo inscreve-se nessa linha de preocupações, tendo como objetivo comparar as sete maiores frequências de morbidade hospitalar em idososno ano de 2005 com o ano de 1994, procurando contribuir para consolidar o conhe- cimento sobre as necessidades da população em foco.

M aterial emétodos

A presente pesquisa desenvolveu um estudo de avaliação das informações obtidas na base de dados do Datasus, do Ministério da Saúde, sobre a morbidade hospitalar nos anos de 2005 e de 1994 em idosos. Em sua estruturação, foram consideradas as etapas especificadas por Fletcher efletcher ${ }^{34}$.

Para a avaliação das internações hospitalares estudadas, foram comparados os anos de 2005 e 1994, sendo utilizada como unidade geográfica de análise o Brasil. Adicionalmente, procedeu-se ao cál culo das taxas de internações hospitalares brutas e ajustadas para as sete maiores frequências.

0 instrumento da coleta de dados foi o Datasus, que contém um sistema de informações em saúde disponível pelal nternet. Os dados deinternações hospital ares foram originados do Sistema de Informações H ospitalares (SIH/M S). Para verificar as morbidades hospitalares, consultou-se o website http://www.datasus.gov.br, onde é possível descobrir tanto a distribuição de internações por faixa etária como também a por sexo ${ }^{35}$.

No Brasil, o Sistema de Informações H ospitalares $(\mathrm{SIH})^{36}$ representa uma fonte de dados de grande importância para o conhecimento do perfil epidemiológico da morbidade hospitalar, pois diversos trabalhos na área de idosos, como a Política Nacional do Idoso, tiveram como fonte a utilização dos dados do Datasus. Veras ${ }^{6}, \mathrm{Ca}$ marano ${ }^{9}$, Lima-Costa et al. ${ }^{37}$ eO liveira et al. ${ }^{7}$ utilizaram-se também das taxas do Datasus.

A utilização do Datasus para outros trabaIhos em áreas de não idosos também se verifica no caso do IBGE ${ }^{38}$ ena autoinstrução em análise de situações da endemia de hanseníase ${ }^{39}$, por exemplo. Desse modo, fica claro o nível de aceitação dessa fonte pela comunidade científica, com o resultado dessas pesquisas revertendo em subsídios para o próprio SUS.

A equipe do Datasus desenvolveu ainda um sistema de informação geográfica (SIG) de fácil utilização para acesso e visualização do cálculo de estatísticas básicas para dados de saúde, disponível na Internet: o TABWIN ${ }^{36}$.

Deve-se ressaltar que, para a realização da avaliação e da comparação, tem-se que ir além da estatística convencional e da mera visualização de dados. É preciso acoplar, aos SIGs tradicionais, as ferramentas de tratamento dos 
dados. Como exemplo, a seleção da população idosa e do ano para o estudo.

Os sujeitos selecionados atendiam ao seguinte critério de inclusão: ter 60 anos de idade ou mais, no ano de 1994 e no ano de 2005.

Inicialmente, foram calculadas as taxas específicas de internação em 1994 e em 2005. Em seguida, selecionaram-se as sete doenças com as maiores frequências de 2005, para compor um quadro comparativo com o ano de 1994. As taxas de internações com as sete maiores frequências foram ajustadas, com seus respectivos intervalos de confiança de $95 \%$. As taxas foram ajustadas empregando-se o método direto de padronização ${ }^{40}$, utilizando-se como referência a população mundial| ${ }^{28}$ e o Censo Demográfico da População Residente- Brasil ${ }^{38}$. 0 método direto de padronização permite que comparações entre períodos ou entre locais possam ser realizadas com o ajuste para possíveis diferenças na estrutura das populações que estão sendo comparadas. Os interval os de confiança de $95 \%$ das taxas padronizadas foram calculados tomando por base 0 erro-padrão para proporções em amostras estratificadas ${ }^{41}$. As análises foram realizadas empregando o programa Epidat 3.142.

Este estudo faz parte de uma pesquisa maior em que os procedimentos de regularização no Comitê de Ética em Pesquisa do Hospital Universitário Pedro Ernesto (Hupe) da Universidade do Estado do Rio de Janeiro (U erj) foram realizados e aprovados (1.607 - Consel ho de Ensino, Pesquisa e Extensão-Cepe/Hupe) conforme a Resolução $n^{\circ} 196$, sobre pesquisa envolvendo seres humanos, de 10 de outubro de 1996, do Conselho Nacional de Saúde.

\section{Resultados}

0 total de morbidade hospitalar encontrada nos indivíduos com 60 anos ou mais foi de 2.539 .067 registros no ano de 1994 e 2.224.147 no ano de 2005, que deram origem à Tabela 1.

$\mathrm{Na}$ segunda fase da pesquisa, na Tabela 1 foram considerados apenas os sete maiores valores de frequência de morbidade hospitalar apresentados em 2005, para serem comparadas com os valores apresentados em 1994 para as mesmas morbidades, dando origem às Tabelas 2 e 3 e ao Gráfico 1.

\section{Discussão}

Tendo como referência as taxas ajustadas da Tabela 3 do ano de2005, tanto por faixa etária quanto por sexo, foram encontradas as sete maiores frequências de morbidade hospitalar nos idosos indicadas a seguir.

As primeiras doenças que se destacaram em 2005 (28\%) com maior número de casos foram as do aparelho circulatório, resultado compatível com a literatura da área. Esse panorama não se modificou em comparação com o ano de 1994 (32\%), pois elas já ocupavam a liderança e se mantiveram na frente das demais morbidades ao longo desse período; porém, em 2005 houveuma redução de $4 \%$ dessas taxas, o que sugereum efeito dos programas iniciais de combate à hipertensão. Esta redução é significativa e estimula o investimento, de forma mais intensiva, em programas preventivos e informativos nessa área.

As segundas doenças encontradas em maior número nas internações no ano de 2005 (17\%) foram as do aparelho respiratório, que também diminuíram 2\% em comparação com 1994 (19\%), porém mantiveram essa mesma classificação desde 1994. Essa redução de 1\% pode estar associada ao programa de vacinação dos idosos (influenza) e também à prática de estimular a redução da internação, concorrendo para diminuir os efeitos da infecção hospitalar e da iatrogenia, pois, uma vez acamados, os idosos ficam predispostos aos acometimentos pulmonares.

As terceiras doenças em maior número encontradas em 2005 (10\%) foram as do aparelho digestivo, que aumentaram $2 \%$ em comparação com 1994 (8\%), mantendo a mesma tendência de sua classificação até 2005, pois em 1994 elas também ocupavam o terceiro lugar. Esse aumento pode estar relacionado ao aumento de doenças relacionadas ao consumo em demasia de algumas medicações (antiinflamatórios), que em geral afetam a parte digestiva, ecom o aparecimento maior de úlceras e gastrite. Problemas dietéticos (má alimentação) também são uma característica dos grandes centrosurbanos, ondesevivencia a falta de tempo, e da cultura moderna, que leva ao consumo exagerado de fast-food e dos alimentos em conserva, que prejudicam o funcionamento intestinal, o qual já é debilitado no idoso.

A quarta doença é a neoplasia, que merece um preocupação maior, pois saiu de uma classificação de 60 lugar em 1994 para 0 4 lugar em $2005(8 \%)$, com um aumento de 4\%, representando o dobro de 1994 (4\%). Isto demonstra ser ela um ponto importante a se pesquisar e sinali- 
zar, tanto para o governo quanto para a sociedade. Recomenda-se, por medida de precaução, informar os serviços de saúde dos tipos de neopla- sia mais prevalentes, no sentido de alertar o profissional de saúde para estar atento à detectação desse mal da forma mais precoce possível, evi-

Tabela 1. M orbidade hospitalar do SUS - 60 anos ou mais - 2005 e 1994.

\begin{tabular}{lrr}
\hline \multicolumn{1}{c}{ Período: 2005 } & \multicolumn{2}{c}{ Total } \\
\hline Capítulo CID-10 & & 159.855 \\
I. Algumas doenças infecciosas e parasitárias & $7,19 \%$ & 179.946 \\
II. N eoplasias (tumores) & $8,09 \%$ & 19.522 \\
III. Doenças sangue órgãos hemat e transt imunitár & $0,88 \%$ & 112.172 \\
IV. Doenças endócrinas nutricionais e metabólicas & $5,04 \%$ & 21.126 \\
V. Transtornos mentais e comportamentais & $0,95 \%$ & 41.805 \\
VI. Doenças do sistema nervoso & $1,88 \%$ & 27.857 \\
VII. Doenças do olho e anexos & $1,25 \%$ & 498 \\
VIII. Doenças do ouvido e da apófise mastóide & $0,02 \%$ & 642.983 \\
IX. Doenças do aparelho circulatório & $28,91 \%$ & 393.638 \\
X. Doenças do aparelho respiratório & $17,70 \%$ & 227.441 \\
XI. Doenças do aparelho digestivo & $10,23 \%$ & 31.827 \\
XII. Doenças da pele e do tecido subcutâneo & $1,43 \%$ & 56.189 \\
XIII. Doenças sist. osteomuscular e tec conjuntivo & $2,53 \%$ & 136.199 \\
XIV. Doenças do aparelho geniturinário & $6,12 \%$ & 2.412 \\
XV. Gravidez parto e puerpério & $0,11 \%$ & 66 \\
XVI. Algumas afec. originadas no período perinatal & $0,00 \%$ & 5.357 \\
XVII. Malf. cong. D eformid. e anomalias cromossômicas & $0,24 \%$ & 39.885 \\
XVIII. Sint. sinais e achad. anorm. ex. clín. e laborat. & $1,79 \%$ & 112.798 \\
XIX. Lesões enven. e alg. out. conseq. causas externas & $5,07 \%$ & 303 \\
XX. Causas externas de morbidade e mortalidade & $0,01 \%$ & 12.268 \\
XXI. Contatos com serviços de saúde & $0,55 \%$ & 2.224 .147 \\
Total & $99,80 \%$ & \\
\hline & & Total \\
\hline
\end{tabular}

\section{Capítulo CID-9}

$\begin{array}{lrr}\text { I. Doenças infecciosas e parasitárias } & 7,15 \% & 175.545 \\ \text { II. N eoplasmas } & 4,64 \% & 113.904 \\ \text { III. Doenças glând. endócr. da nutr. e do metab. e transt. imun. } & 4,49 \% & 110.228 \\ \text { IV. Doenças do sangue e dos órgãos hematopoéticos } & 0,69 \% & 16.943 \\ \text { V. Transtornos mentais } & 1,56 \% & 38.403 \\ \text { VI. Doenças sistema nervoso órgãos dos sentidos } & 4,16 \% & 102.167 \\ \text { VII. Doenças do aparelho circulatório } & 32,84 \% & 806.016 \\ \text { VIII. Doenças do aparelho respiratório } & 19,04 \% & 467.369 \\ \text { IX. Doenças do aparelho digestivo } & 8,87 \% & 217.626 \\ \text { X. Doenças do aparelho geniturinário } & 6,23 \% & 152.876 \\ \text { XI. Complicações da gravidez, parto e puerpério } & 0,04 \% & 996 \\ \text { XII. Doenças da pele e tecido celular subcutâneo } & 1,29 \% & 31.542 \\ \text { XIII. Doenças sistema osteomusc. e tecido conjuntivo } & 2,26 \% & 55.371 \\ \text { XIV. Anomalias congênitas } & 0,19 \% & 4.541 \\ \text { XV. Algumas afecções originadas período perinatal } & 0,04 \% & 1.028 \\ \text { XVI. Sintomas, sinais e afecções mal definidas } & 1,79 \% & 43.854 \\ \text { XVII. Lesões e envenenamentos } & 4,51 \% & 110.790 \\ \text { Class. supl. fatores oport. contato serviços saúde } & 0,19 \% & 4.619 \\ \text { CID não especificado ou inválido } & 0,03 \% & 850 \\ \text { Total } & 99,9 \% & 2.454 .668\end{array}$

Fonte: M orbidade hospitalar do SU S por faixa etária. In: Brasil, M inistério da Saúde, D atasus, $2006{ }^{43}$. 
tando o aumento desses números. Também se pressupõequeesse resultado estácompatível com o aumento observado das neoplasias do sexo masculino em relação às do sexo feminino em 2005, quando comparado ao ano de 1994 - o que pode ser atribuído ao aumento de neoplasias em próstata nos últimos anos.

Em quinto lugar temos as doenças infecciosas e parasitárias, que em 2005 registraram 7\% e mantiveram basicamenteo mesmo percentual em 1994 (7\%), porém desceram de classificação em 2005, pois em 1994 ocupavam o quarto lugar.
Isto se deu em virtude do aumento das neoplasias, empurrando as doenças infecciosas e parasitárias para o quinto lugar. Tal resultado carece de maiores pesquisas, pois pressupõe-se que em termos de doen ças infecciosas e parasitárias praticamente nada mudou, trazen do um questionamento sobre a eficácia das campanhas e programas nessa área para os idosos, programas e campanhas que devem ser revistos. Fica evidenteque, no Brasil, mesmo tendo mudado o perfil epidemiológico das doenças crônicas, ainda não setem medidas de forma abrangente que possam dimi-

Tabela 2. As sete maiores frequências de morbidade hospitalar do SUS (60 anos ou mais) por faixa etária - 2005 e 1994.

\begin{tabular}{|c|c|c|c|c|c|}
\hline Período: 2005 & \multicolumn{5}{|c|}{ Período: 1994} \\
\hline Capítulo CID-10 & \multicolumn{2}{|c|}{ Total } & Capítulo CID-9 & \multicolumn{2}{|c|}{ Total } \\
\hline I. Algumas doenças infecciosas e & $7,19 \%$ & 159.855 & I. Doenças infecciosas e parasitárias & $7,15 \%$ & \\
\hline parasitárias & & & II. Neoplasmas & $4,64 \%$ & 113.904 \\
\hline II. N eoplasias (tumores) & $8,09 \%$ & 179.946 & VII. Doenças do aparelho & $32,84 \%$ & 806.016 \\
\hline IX. Doenças do aparelho circulatório & $28,91 \%$ & 642.983 & circulatório & & \\
\hline X. D oenças do aparelho respiratório & $17,70 \%$ & 393.638 & VIII. Doenças do aparelho & $19,04 \%$ & 467.369 \\
\hline XI. Doenças do aparelho digestivo & $10,23 \%$ & 227.441 & respiratório & & \\
\hline XIV. Doenças do aparelho & $6,12 \%$ & 136.199 & IX. Doenças do aparelho digestivo & $8,87 \%$ & 217.626 \\
\hline geniturinário & & & X. Doenças do aparelho & $6,23 \%$ & 152.876 \\
\hline XIX. Lesões enven. e alg. out. & $5,07 \%$ & 112.798 & geniturinário & & \\
\hline conseq. causas externas & & & XVII. Lesões e envenenamentos & $4,51 \%$ & 110.790 \\
\hline Total & 99,9 & 2.224 .147 & Total & $99,9 \%$ & 2.454 .668 \\
\hline
\end{tabular}

Fonte: M orbidade hospitalar do SU S por faixa etária. In: Brasil, M inistério da Saúde, Datasus, $2006^{43}$.

Tabela 3. Taxa de morbidade hospitalar ${ }^{*}$ por internação no Brasil, segundo o ano.

\begin{tabular}{|c|c|c|c|c|c|}
\hline Doenças & População & $\begin{array}{l}\text { Percentual } \\
\text { do total }\end{array}$ & Taxa bruta & $\begin{array}{c}\text { Taxa } \\
\text { ajustada(1) }^{(1)}\end{array}$ & IC $(95 \%)^{* *}$ \\
\hline \multirow{2}{*}{$\begin{array}{l}\text { I. Algumas doenças infecciosas } \\
\text { eparasitárias }\end{array}$} & 2005 & $07,187 \%$ & 7187,2853 & 7213,7447 & $7178,34527249,1441$ \\
\hline & 1994 & $07,151 \%$ & 7151,6687 & 7173,9973 & $7140,41447207,5802$ \\
\hline \multirow[t]{2}{*}{ II. N eoplasias (tumores) } & 2005 & $08,090 \%$ & 8090,6024 & 8056,3791 & $8019,11648093,6418$ \\
\hline & 1994 & $04,640 \%$ & 4640,4386 & 4634,8379 & $4607,90264661,7731$ \\
\hline \multirow[t]{2}{*}{ IX. Doenças do aparelho circulatório } & 2005 & $28,909 \%$ & 28909,2034 & 28925,3848 & $28854,608928996,1607$ \\
\hline & 1994 & $32,836 \%$ & 32836,9811 & 32852,7933 & $32781,021232924,5654$ \\
\hline \multirow[t]{2}{*}{ X. Doenças do aparelho respiratório } & & & 17698,4231 & 17668,3147 & $17613,062017723,5674$ \\
\hline & 1994 & $19,040 \%$ & 19040,5341 & 19019,6283 & $18965,061619074,1951$ \\
\hline \multirow[t]{2}{*}{ XI. Doenças do aparelho digestivo } & 2005 & $10,226 \%$ & 10225,9484 & 10194,9119 & $10152,9691 \quad 10236,8547$ \\
\hline & 1994 & $08,865 \%$ & 8866,1006 & 8841,4458 & $8804,2733 \quad 8878,6184$ \\
\hline \multirow{2}{*}{$\begin{array}{l}\text { XIV. Doenças do aparelho } \\
\text { geniturinário }\end{array}$} & 2005 & $06,123 \%$ & 6123,5914 & 6103,7932 & $6071,3426 \quad 6136,2438$ \\
\hline & & & 6228,1683 & 6189,1955 & $6158,1488 \quad 6220,2422$ \\
\hline XIX. Lesões enven. e alg. out. & 2005 & $05,071 \%$ & 5071,4525 & 5087,28304 & $5057,5636 \quad 5117,0023$ \\
\hline conseq. causas externas & 1994 & $04,513 \%$ & 4513,6142 & 519,9183 & $4493,2846 \quad 4546,5520$ \\
\hline
\end{tabular}

(*) Valores por 100.000; (**) Intervalo de confiança (95\%) da taxa ajustada; (1) População padrão do Brasil proposta pelo Censo Demográfico². 


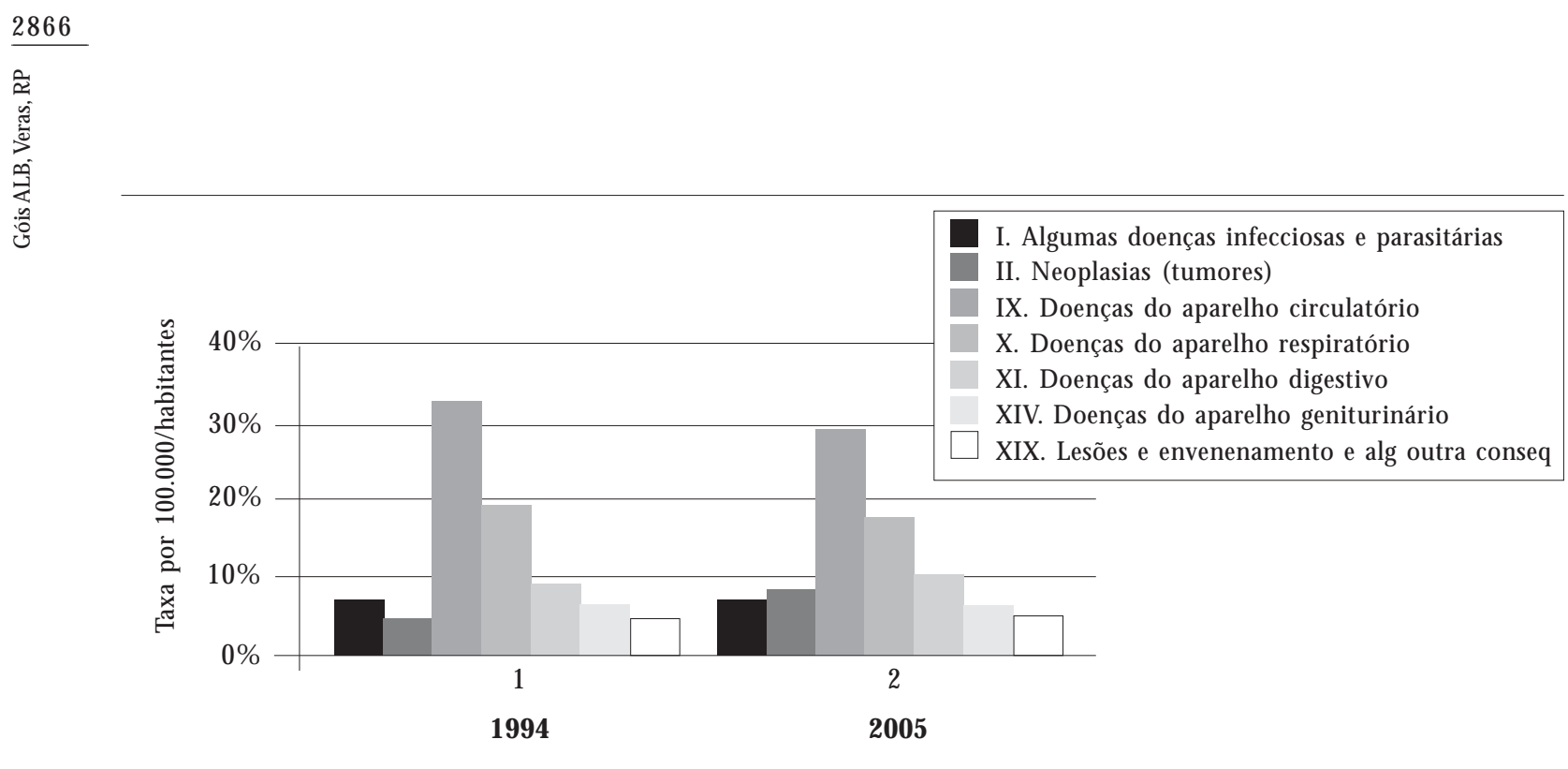

Gráfico 1. As sete maiores frequências de morbidade hospitalar registradas em 2005 nos idosos, comparadas com os dados registrados em 1994.

nuir esses números, demonstrando que o perfil epidemiológico anterior ainda é um problema para o país. Ressalta-se também que esse problema pode estar ligado à falta de saneamento básico na maioria dos municípios brasileiros.

Em sexto lugar aparecem as doenças geniturinárias: em 2005 (6\%), elas mantiveram basicamenteo mesmo percentual do ano de 1994 (6\%), porém desceram na classificação geral em 2005, pois em 1994 ocupavam o quarto lugar. Isto se deu devido ao aumento das neoplasias, que empurraram as doenças geniturinárias para o sexto lugar. Esteresultado demonstra que praticamente nada mudou, podendo sugerir novos estudos ea revisão de campanhas e programas nessa área. Percebe-se que essas doen ças podem estar relacionadas à fragilidade dos idosos e à iatrogenia, em que o idoso, já fragilizado por uma doença principal, estaria propenso às infecções de maneira geral, e em particular às do aparelho geniturinário, que estão intimamenteligadas à infecção hospitalar.

No sétimo lugar temos as lesões e envenenamentos, que em 2005 (5\%) aumentaram 1\% em comparação com 1994 (4\%), descendo sua classificação geral em 2005, pois em 1994 ocupavam o sexto lugar. 0 aumento dessas taxas sugere novos estudos, e coloca-se em questão a influência das quedas nos idosos como um dos fatores de maior destaque para essa morbidade. Devese também incentivar a importância dos cuidados relacionados após a alta, para diminuir a taxa de reinternação.
As doenças endócrinas nutricionais e metabólicas, apesar de não aparecerem entre as sete primeiras, merecem ser comentadas porque, embora ocupem o 80 lugar em 2005, seu percentual era de 4\% em 1994 eaumentou para 5\% em 2005. Este aumento de $1 \%$ nos idosos é um dos motivos de estudos recentes e de preocupação atual, devido ao aumento da obesidade na população em geral e da diabetes, que está ocorrendo em proporções cada vez maiores e em faixas etárias cada vez mais baixas.

De forma mais abrangente, ao se comparar o comportamento das taxas ajustadas de morbidade hospitalar dos anos de 1994 e 2005 em relação à evolução do tempo, pode-severificar quehouve um padrão de comportamento decrescente nas doenças do aparelho circulatório e do aparelho respiratório; quehouveum padrão decomportamento crescente nas doen ças do aparel ho digestivo, nas neoplasias e nas lesões eenvenenamentos; eque houve um padrão de comportamento praticamenteinalterado nas doenças geniturináriase nas doenças infecciosas e parasitárias.

\section{Conclusão}

A respeito das estruturas de atendimento, tendese a recomendar uma estratégia integrada da família com o hospital geriátrico e, para os portadores de incapacidade funcional severa, a assistência domiciliar. A assistência domiciliar não pode ser considerada um substituto dos trata- 
mentos hospitalares, porém necessita ser acoplada ao sistema de saúde, permitindo assim que se limite o recurso à hospitalização dos idosos. No entanto, nada disso terá efeito se a falta de difusão do conhecimento geriátrico junto aos profissionais de saúde não for resolvida. Decisivamente, a dificuldade na abordagem terapêutica do paciente idoso éo queleva a um atendimento de baixa qualidade e à iatrogenia.

0 período pós-hospitalização é fonte de preocupação nas pesquisas recentes, pois normalmente o idoso não consegue retornar às suas atividades diárias anteriores à internação, que contribui para aumentar o número de idosos com limitações e incapacidades geradas pelas doenças crônicas no envelhecimento. Esse quadro é agravado pela falta de encaminhamento e acesso à reabilitação precoce do idoso após a sua hospitalização. Isto é preocupante pela perspectiva do aumento desses casos, o que se reflete diretamente na saúde pública.

M edianteos resultados desta pesquisa, podese concluir que, no Brasil, a morbidade hospitalar em idosos mantém o predomínio das doenças do aparelho circulatório, ao mesmo tempo que se observa a não redução das doenças infecciosas eparasitárias; eainda sinaliza-se o aumento recente e acentuado das neoplasias.

Em se tratando de saúde pública, sugere-se que as doenças com presença crescente ou inalterada nos registros do Datasus sejam alvo de maiores pesquisas eatenções. Sinaliza-se, em especial, o aumento acentuado da neoplasia ea não redução das doenças infecciosas e parasitárias. Com relação às medidas que visem diminuir as morbidades hospitalares prevalentes, percebe-se a necessidade urgente de se ampliarem as assistências domiciliares para melhorar a saúde dos idosos e, consequentemente, para se limitar 0 recurso à hospitalização.

A comparação das taxas de morbidade hospitalar padronizadas, observadas em nosso estudo, corroboram os resultados de pesquisas semel hantes da área, mas mesmo assim essa afirmação deve ser feita com cautela: os valores encontrados podem variar de acordo com a população de referência e das faixas etárias utilizadas no processo de padronização. Além disto, outros fatores devem ser considerados, entre eles 0 fato de o SIH/SUS ter sido concebido para operar o sistema de pagamento de internação nos hospitais contratados pelo M inistério da Previdência. Em 1986, essa incumbência foi estendida aos hospitais filantrópicos; em 1987, aos universitários e de ensino; e, em 1991, aos hospitais públicos municipais, estaduais e federais (administração indireta e outros ministérios). M ediante a consulta do manual do $\mathrm{SIH}^{36}$, verificou-se queestereúneinformações apenas sobre60-70\% das internações hospitalares realizadas no país. 0 documento básico éa Autorização de Internação H ospitalar (AIH), que habilita a internação do paciente e gera valores para pagamento. 0 sistema disponibiliza dados de forma sistemática, com defasagem de apenas dois meses a partir da data de internação.

A abrangência do sistema está limitada às internações no âmbito do SUS, excluindo, portanto, as que são custeadas diretamente ou cobertas por seguro-saúde. Eventuais reinternações e transferências do mesmo paciente a outros hospitais também não são identificadas, o que pode resultar em contagem cumulativa. Além disso, foram verificados nesta pesquisa (ver Tabelas $1 \mathrm{e}$ 2) números de morbidades hospitalares nas doenças de afecções originadas no período perinatal e nas doenças de complicações de gravidez, parto e puerpério, sendo questionável o aparecimento desses número nessas doenças em se tratando de idosos e pacientes do sexo masculino.

Finalmente, recomenda-se que os estudos de comparações entre períodos estejam entre as ferramentas de uso na gestão, concorrendo assim para a melhoria da saúde dos idosos do Brasil. 


\section{Colaboradores}

ALB Góis participou na escolha do tema e na execução da introdução, da revisão da literatura, do Resumo, do Abstract e da conclusão. Participou em parte na construção da metodologia escolhida e da discussão dos resultados. RP Veras participou na definição da metodologia ena discussão dos resultados, além da orientação geral do trabalho.

\section{Agradecimentos}

Os autores agradecem o financiamento eo apoio da Fundação Carlos Chagas Filho de Amparo à Pesquisa do Estado do Rio de Janeiro (Faperj), pela bolsa de matrícula 으 2007.0038.3, estruturada pela lei no 1.175 , de 21-07-87, Secretaria do Estado do Rio deJaneiro, Governo do Estado do Rio de Janeiro.

\section{Referências}

1. Santana CM . Aspectos clínicos na prática geriátrica. In: Pereira CU, Andrade Filho A de S. N euroge riatria. Rio de Janeiro: Revinter, 2001. p. 46-50.

2. Instituto Brasileiro de Geografia e Estatística (IBGE). Perfil dos idosos responsáveis pelos domicílios no Brasil. Rio de Janeiro: IBGE; 2002. [acessado $2006 \mathrm{fev}$ 10]. Disponível em: http://www.ibge.gov.br

3. Kalache A, Veras RP, Ramos LR. $O$ envelhecimento da população mundial: um desafio novo. Rev Saude Publica 1987; 21(3):200-210.

4. Ramos LR. A explosão demográfica da terceira idade no Brasil: uma questão de saúde pública. Gerontologia 1993; 1(1):3-8.

5. Veras RP. País jovem com cabelos brancos: a saúde do idoso no Brasil. Rio de Janeiro: Relume Dumará, Uerj; 1994.

6. Veras RP. Terceira idade: gestão contemporânea em saúde. Rio de Janeiro: Relume Dumará, Unati/Uerj; 2002.

7. Oliveira GM M, Klein CH, Silva NAS. Mortalidade por doenças isquêmicas do coração, doenças cerebrovasculares e causas mal definidas nas regiões de saúde do estado do Rio de Janeiro, no período de 1980 a 2000. Rev. Socerj 2005; 8:13-22.

8. Karsch UMS, organizador. Envelhecimento com dependência: revelando cuidadores. São Paulo: Educ; 1998.

9. Camarano AA, organizador. Os novos idosos brasileiros: muito além dos 60? Rio de Janeiro: Ipea; 2004.

10. Carvalho-Filho ET. Iatrogenia em pacientes idosos hospitalizados. Rev Saude Publica 1998; 32(1):36-42.

11. Rothschild JM, Bates DW, Leape LL. Preventable medical injuries in older patients. Arch Intern $M$ ed 2000; 160:2717-2728.

12. Veras $R$, Lourenço R. Formação humana em geriatria e gerontologia: uma perspectiva interdisciplinar. Rio de Janeiro: Unati, Uerj; 2006. p. 271-297.

13. Cumming RG, Klineberg RJ. Fall frequency and characteristics and the risk of hip fractures. JAGS 1994; 42:774-778.

14. Rebelatto JR, M orelli JGS. Fisioterapia geriátrica. São Paulo: Manole; 2004.

15. Gross PA, Rapuano C, Adrignolo A, Shaw B. Nosocomial infections: decade specific risk. Infect Control 1983; 4:145-147.

16. Brandeis GH, Morris JN, Nash DJ, Lipsitz LA. The epidemiology and natural history of pressure ulcers in elderly nursing home residents. JAM A 1990; 264:2905-2909.

17. Armstrong O, Bortz P. An integrative review of pressure relief in surgical patients. AORN J 2001; 73(3):645-674. 
18. Perez ED. Pressure ulcers: updated guidelines for treatment and prevention. Geriatrics 1993; 48(1):3944.

19. Saldanha $A L$, Caldas $P C$, organizadores. Saúde do idoso: a arte de cuidar. $2^{\text {a }}$ ed. Rio de Janeiro: Interciência; 2004.

20. Manidi JM, Michel JP. Atividade física para adultos com mais de 55 anos: quadros clínicos e programas de exercícios. São Paulo: Manole; 2001.

21. Santos SPT. Atividade física sob uma perspectiva existencial de vida para o idoso sedentário autônomo: uma reflexão axiológica e fenomenológica [dissertação de mestrado]. Rio de Janeiro: Universidade Castelo Branco; 2002.

22. Moriguchi Y, Moriguchi EH. Biologia geriátrica ilustrada. São Paulo: Fundação BYK; 1988.

23. Almeida NM. A reinserção socioprofissional do idoso no mundo tecnológico [dissertação de mestrado]. Florianópolis: Universidade Federal de Santa Catarina; 2001.

24. Keller SM, Markovitz LJ, Wilder JR, Aufses AHJ. Emergency and elective surgery in patients over age 70. Am Surg 1987; 53:636-640.

25. Doucet J, Capet $C$, J ego A, Trivalle C, Noël D, Chassagne $P$, Bercoff $E$. Les effets indésirables des médicaments chez le sujet âge: épidémiologie et prévention. Presse M ed 1999; 28:1789-1793.

26. Gerety MB, Cornell JE, Plichta DT, Eimer M. Adverse events related to drugs and drug withdrawl in nursing home residents. JAGS 1993; 41:1326-1332.

27. Bates DW, Leape LL, Cullen DJ, Laird N, Petersen LA, Teich JM, Burdick E, Hickey M, Kleefield S, Shea B, Vliet MV, Seger DL. Effect of computerized physician order entry and a team intervention on prevention of serious medication errors. JAM A 1998; 280:1311-1316.

28. Brasil. M inistério da Saúde/SAS/Inca. Câncer no Brasil: dados dos registros de base populacional, 3. Rio de Janeiro: Inca; 2003.

29. Brasil. Ministério da Saúde. Secretaria de Vigilância em Saúde. Departamento de Análise de Situação em Saúde. Saúde Brasil 2005: uma análise da situação de saúde no Brasil. Brasília: Ministério da Saúde; 2005.

30. Mendes W. Home care: uma modalidade de assistência à saúde. Rio de Janeiro: U erj, Unati; 2001.

31. Odenheimer G L. Geriatric neurology. Neurologic Clinics 1998; 16(3):561-567.

32. Góis ALB. 0 valor das condutas e comportamentos motores voltados para indivíduos idosos portadores da Doença de Parkinson [dissertação de mestrado]. Rio de Janeiro: Universidade Castelo Branco; 2005.

33. Brasil. Ministério da Saúde. O SUS de A a Z: garantindo saúde nos municípios. Brasília: Ministério da Saúde; 2006.
34. Fletcher RH, Fletcher SW. Epidemiologia clínica: elementos essenciais. $4^{a}$ ed. Rio de Janeiro: Artmed; 2006.

35. Camargo Jr. KR, Coeli CM. Sistemas de informação e banco de dados em saúde: uma introdução. Rio de Janeiro: IM S/U erj; 2000. (Série Estudos em Saúde Coletiva, 209)

36. Brasil. M inistério da Saúde. Secretaria de Atenção à Saúde (SAS). Departamento de Regulação, Avaliação e Controle (DRAC). Coordenação-Geral de Sistemas de Informação (CGSI). M anual do Sistema de Informações H ospitalares do SUS (SIH/SUS). Brasília: M inistério da Saúde; 2005.

37. Lima-Costa MF, Guerra HL, Barreto SM, Guimarães RM. Diagnóstico da situação de saúde da população idosa brasileira: um estudo da mortalidade e das internações hospitalares públicas. Inf Epidemiol SU S 2000; 9:23-41.

38. Instituto Brasileiro de Geografia e Estatística (IBGE). Censo Demográfico 2000. Rio de Janeiro: IBGE; 2000. [acessado 2003 jun 12]. Disponível em: http://www. mpas.gov.br/12_01_18.htm

39. Brasil. M inistério da Saúde. Autoinstrução em análise de situações da endemia de Hanseníase - Base de dados Sinan Hanseníase. [acessado 2006 ago 20]. Disponível em: http://hanseniase.datasus.gov.br

40. Curtin LR, Klein RL. Direct standardization (ageadjusted death rates). H ealth People 2000 - Statistical Notes 1995 2000; 6:1-10.

41. Cochran WG. Sampling techniques. $3^{\text {rd }}$ ed. New York: John Wiley \& Sons; 1977.

42. Epidat. Programa para análise epidemiológica de dados tabulados: versão 3.1. [acessado $2006 \mathrm{mar}$ 22] Disponível em: http://dxsp.sergas.es. Washington: Opas; 2005.

43. Brasil. M inistério da Saúde. Departamento de Informática do SUS (Datasus). Informações de saúde. Morbidade hospitalar. [acessado 2006 abr 20]. Disponível em: http://www.datasus.gov.br.

Artigo apresentado em 18/08/2007

Aprovado em 04/03/2008

Versão final apresentada em 10/04/2008 